Image Analysis

Elsevier Editorial System(tm) for Medical

Manuscript Draft

Manuscript Number:

Title: Prediction of final infarct volume from native CT perfusion and treatment parameters using deep learning

Article Type: Research Paper

Keywords: Stroke; CT Perfusion; Final Infarct Prediction; Deep Learning

Corresponding Author: Dr. David Robben,

Corresponding Author's Institution: KU Leuven

First Author: David Robben

Order of Authors: David Robben; Anna Boers, PhD; Henk Marquering, PhD; Lucianne Langezaal, MD; Yvo Roos, MD PhD; Robert van Oostenbrugge, MD PhD; Wim van Zwam, MD PhD; Diederik Dippel, MD, PhD; Charles Majoie, MD, PhD; Aad van der Lugt, MD, PhD; Robin Lemmens, MD, PhD; Paul Suetens, Phd

Abstract: CT Perfusion (CTP) imaging has gained importance in the diagnosis of acute stroke.

Conventional perfusion analysis performs a deconvolution of the measurements and thresholds the perfusion parameters to determine the tissue status.

We pursue a data-driven and deconvolution-free approach, where a deep neural network learns to predict the final infarct volume directly from the native CTP images and metadata such as the time parameters and treatment.

This would allow clinicians to simulate various treatments and gain insight into predicted tissue status over time.

We demonstrate on a multicenter dataset that our approach is able to predict the final infarct and effectively uses the metadata.

An ablation study shows that using the native CTP measurements instead of the deconvolved measurements improves the prediction. 


\title{
Prediction of final infarct volume from native CT perfusion and treatment parameters using deep learning
}

\author{
David Robben a,b,c,*, Anna M.M. Boers ${ }^{\mathrm{d}}$, Henk A. Marquering ${ }^{\mathrm{d}}$, Lucianne L.C.M. Langezaal ${ }^{\mathrm{h}}$, Yvo B.W.E.M. Roos ${ }^{\mathrm{d}}$, Robert J. van \\ Oostenbrugge $^{\mathrm{f}}$, Wim H. van Zwam ${ }^{\mathrm{f}}$, Diederik W.J. Dippel ${ }^{\mathrm{g}}$, Charles B.L.M. Majoie ${ }^{\mathrm{e}}$, Aad van der Lugt $^{\mathrm{g}}$, Robin Lemmens ${ }^{\mathrm{i}, \mathrm{j}, \mathrm{k}}$, \\ Paul Suetens ${ }^{\mathrm{a}, \mathrm{b}}$ \\ ${ }^{a}$ Medical Imaging Research Center (MIRC), KU Leuven, Leuven, Belgium \\ ${ }^{b}$ Medical Image Computing (MIC), ESAT-PSI, Department of Electrical Engineering, KU Leuven, Leuven, Belgium \\ ${ }^{c}$ Icometrix, Leuven, Belgium \\ ${ }^{d}$ Amsterdam UMC, Amsterdam, the Netherlands \\ ${ }^{e}$ Amsterdam UMC, location AMC, Amsterdam, the Netherlands; Department of Radiology and Nuclear Medicine

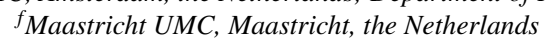 \\ ${ }^{g}$ Erasmus MC, Rotterdam, the Netherlands \\ ${ }^{h}$ St. Antonius Ziekenhuis, Nieuwegein, the Netherlands \\ ${ }^{i}$ Department of Neurosciences, Experimental Neurology, and Leuven Brain Institute (LBI), KU Leuven - University of Leuven, Leuven, Belgium \\ ${ }^{j}$ VIB, Center for Brain $\mathcal{E}$ Disease Research, Laboratory of Neurobiology, Leuven, Belgium \\ ${ }^{k}$ University Hospitals Leuven, Department of Neurology, Leuven, Belgium
}

\begin{abstract}
CT Perfusion (CTP) imaging has gained importance in the diagnosis of acute stroke. Conventional perfusion analysis performs a deconvolution of the measurements and thresholds the perfusion parameters to determine the tissue status. We pursue a data-driven and deconvolution-free approach, where a deep neural network learns to predict the final infarct volume directly from the native CTP images and metadata such as the time parameters and treatment. This would allow clinicians to simulate various treatments and gain insight into predicted tissue status over time. We demonstrate on a multicenter dataset that our approach is able to predict the final infarct and effectively uses the metadata. An ablation study shows that using the native CTP measurements instead of the deconvolved measurements improves the prediction.
\end{abstract}

Keywords: Stroke, CT Perfusion, Final Infarct Prediction, Deep Learning

\section{Introduction}

Ischemic stroke, a major cause of mortality and disability worldwide, is an acute disease where the blood supply to the brain is hindered due to the occlusion of an artery. Due to 5 the reduction in perfusion, neuronal functioning is impaired and if perfusion is not re-established, brain tissue becomes irreversibly damaged. Acute treatment aims at reopening the blocked artery through treatment with intra-venous trombolytics and/or mechanical thrombectomy. Since both treatment op10 tions entail considerable costs and side effects (e.g. increased risk of haemorrhage), selection of patients who might benefit is important. In early studies, patient selection was mainly based on the time since stroke onset; e.g. intravenous trombolysis with tPA is shown to be beneficial within 4.5 hours 15 (Hacke et al. 2008). Recent studies typically select patients based on both time parameters and advanced neuroimaging, allowing patient-specific assessment of the benefits and risk of treatment.

One commonly used imaging modality for acute stroke diagnosis is CT Perfusion (CTP), which consists of a series of

\footnotetext{
${ }^{*}$ Corresponding author

Email address: david.robben@kuleuven. be (David Robben )
}

3D CT scans acquired after intra-venous injection of contrast agent. The resulting 4D image shows the passage of contrast agent through the brain: in each spatial voxel we have a time-attenuation curve showing the variation of intensity due to the contrast agent. Much research has gone into the quantification of these images, particularly aiming at the estimation of perfusion parameters such as cerebral blood flow (CBF), cerebral blood volume (CBV) and Tmax. The most used approach for perfusion analysis works as follows (Fieselmann et al. 2011). First the time-attenuation curves are converted to time-concentration curves that show the concentration of contrast agent over time. In CTP, there is a linear relationship between the change in attenuation and the concentration. Then an arterial input function (AIF) is determined: this is the timeconcentration curve in one of the large feeding arteries of the brain. Subsequently, the time-concentration curve in each voxel is deconvolved with the AIF, resulting in deconvolved timeconcentration curves. These curves correspond to what would have been measured if the contrast bolus was infinitely short and infinitely concentrated in the feeding artery - indeed, these curves are impulse response functions (IRF). As such, the deconvolved time-concentration curves are no longer influenced by the contrast injection protocol or the cardiac system of the 
particular patient. Finally, under some reasonable assumptions, example $\mathrm{CBF} \propto \max _{t} \operatorname{IRF}(t)$ and Tmax $=\operatorname{argmax}_{t} \operatorname{IRF}(t)$.

The deconvolution operation plays a central role in current perfusion analysis. However, deconvolution depends crucially on the accurate selection of an AIF. Additionally, deconvolution ${ }_{105}$ ${ }_{50}$ is a mathematically ill-posed problem and that is problematic given the low signal to noise ratio of perfusion images (Fieselmann et al. 2011). Perfusion analysis software accounts for this by preprocessing the images and by regularizing the deconvolution. The preprocessing mainly aims at reducing the

55 noise through motion correction, temporal and spatial smoothing, and possibly spatial downsampling. The regularization of the deconvolution suppresses the high frequency signal in the reconstructed impulse response function. This can be done in singular value decomposition (SVD) based deconvolution by

60 regularizing the singular values, e.g. using Tikhonov regularization. Nevertheless, the deconvolution-based perfusion parameters remain noise sensitive and research for improved algorithms (Boutelier et al., 2012) or even deconvolution-free summary parameters (Meijs et al., 2016) remains ongoing.

65 The resulting perfusion parameters are used to assess the tissue status of the brain where - apart from healthy tissue - two types are distinguished. Tissue that is already irreversibly damaged is called the infarct core. Tissue that is at risk, i.e. tissue that will undergo infarction if not eventually reperfused,

70 is called the penumbra. The combination of infarct core and ${ }^{125}$ penumbra is called the perfusion lesion. In current clinical practice, the perfusion lesion and core are determined by thresholding the perfusion parameters. The optimal choice of perfusion parameters and thresholds depends on the deconvolution 75 method (Bivard et al., 2013).

Knowledge about the volumes of the core and the penumbra ${ }_{130}$ is of great clinical importance, as they are used to determine which treatment a patient should get (Albers, 2018, Nogueira et al. 2018). However, the method that is used to analyze the CTP images plays an important role in the accuracy of these volume estimations (Fahmi et al., 2012, Bivard et al., 2013). ${ }_{135}$ Although great progress has been made in the previous years, there is room and need for improvement.

The binary distinction between core and penumbra is some85 what artificial: as time passes, the core will become larger, as more and more penumbral tissue becomes irreversibly dam-140 aged. The infarct growth rate differs between patients (Wheeler et al. 2015, Guenego et al., 2018). It depends on factors such as the location of the thrombus and the patient's vascular con-

90 nectivity: there is considerable inter-subject variation in the amount of vascular redundancy and hence in the amount col-145 lateral blood supply to the affected region (Liebeskind, 2003). Knowledge about the growth rate of the core is of clinical importance, as it allows assessment of the relevance of transfer-

95 ring a patient to a comprehensive stroke center depending on transport time. Hence it is valuable to not only predict core ${ }_{150}$ and penumbra from an acute CTP scan, but also how the core would evolve over time. Additionally, not every mechanical thrombectomy procedure achieves complete reperfusion. Al- ter (Kleine et al., 2017), it is interesting to take this into account in the model as it might give interventional radiologists information about the added value of an additional attempt after a partial recanalization.

\subsection{Contributions}

In this paper we propose to train a deep neural network to predict the final infarct from acute CTP images. The prediction takes into account not only the CTP measurements, but also the treatment parameters such as the time-to-recanalization and the completeness of recanalization. Once the network is trained, it can make predictions for new patients based on hypothetical treatment schemes. This allows to predict the ischemic core at baseline (i.e. the predicted final infarct in case of an immediate perfect recanalization), the perfusion lesion (i.e. the predicted final infarct in case of no recanalization) or any intermediate scenario. The latter can be relevant if e.g. we first need to transfer the patient and achieve recanalization at a particular later time point. This provides clinicians with additional information on the impact of potential treatment options. We show that our method takes this information effectively into account resulting in better predictions.

Another contribution of this work is that the predictions are made directly from the native CTP images while no explicit deconvolution or calculation of perfusion parameters is performed. We follow a true end-to-end learning approach and hence avoid the problematic deconvolution. We evaluate our approach on the data of the MR CLEAN trial and show the contributions of the various components of the method.

\subsection{Related work}

Estimation of tissue status and prediction of the final infarct based on perfusion measurements is a vast research domain. Early work aimed mainly at finding thresholds for various perfusion parameters that correlated well with the final infarct in patients with recanalization (with the final infarct closely related to the core) and patients without recanalization (final infarct equal to the perfusion lesion) (Wintermark et al., 2006, Bivard et al. 2013). These approaches are currently used in clinical practice.

Various works have suggested to use more advanced machine learning techniques to predict core and penumbra. Most of them use MR imaging which usually consists of two modalities: MR Perfusion, which is similar to CTP, and diffusion weighted imaging (DWI) (Wu et al., 2001; Scalzo et al., 2012; McKinley et al., 2017; Nielsen et al., 2018). The latter modality gives a clear signal regarding the viability of tissue and is considered the golden standard to identify ischemic tissue, making the prediction of final infarct easier than using only perfusion information. With the advent of mechanical thrombectomy, accurate determination of the time of recanalization became possible and this parameter was introduced in models. D'Esterre et al. (2015) investigated the influence of the time between onset and imaging and the time between imaging and recanalization, finding that the former does not and the latter does influence the optimal perfusion parameters thresholds to determine 
155 the final infarct. Kemmling et al. (2015) proposed a multivariate generalized linear model that predicts the final infarct in a voxelwise fashion based on CTP perfusion parameters and clinical parameters including a binary recanalization status and the onset-to-recanalization duration. It was trained and cross160 validated on 161 subjects that underwent CTP imaging and mechanical thrombectomy. In a similar vein, the ISLES 2016 \& 2017 challenges (Winzeck et al., 2018) invited researchers to evaluate methods that predict the final infarct based on acute DWI and MR Perfusion imaging. The organizers provided pub-

165 licly available training data and a blinded test set for the validation. The challenge showed the competitive advantage of deep neural networks.

All approaches discussed so far depend on deconvolution as $_{220}$ a first step, but there has been some work to avoid explicit deconvolution. On one hand we can distinguish alternative perfusion parameters that do not require deconvolution, such as the first moment of the time-concentration curve (Christensen et al. 2009). On the other hand, we see approaches that aim at ${ }_{225}$ replacing the traditional deconvolution analysis with a learned alternative (Ho et al., 2016). However, this approach seems to beg the question, since during training the ground truth perfusion parameters are provided by deconvolution analysis. In our approach - starting from the native images and optimizing the network for the best final infarct prediction - the optimal them is inferred directly from the data. The disadvantage of this approach is that those features no longer have a clear physiological interpretation such as the perfusion parameters have, but depending on the application, this might be a price worth paying. Recently Pinto et al. (2018) proposed a method for the ISLES 2017 challenge that ${ }^{235}$ uses, apart from the DWI image and the perfusion parameters, also the native MR perfusion measurements to predict the final infarct. They show that their method benefits from the native measurements, but the obtained results are not as good as other ments, making it hard to assess the added value of the native measurements. The method did not take the reperfusion status or other treatment data into account, presumably because the dataset was fairly homogenous, consisting of mostly early reperfused patients.

\section{Methods}

We propose to use a voxelwise classification approach, where a neural network learns the relation between the final infarct status of a voxel (the output) and the CTP measurements and the metadata (the input). The CTP measurements consists of 255 the time-attenuation curves of that voxel, its neighboring voxels and the voxel of the AIF. The metadata consists of four values: the time between stroke onset and CTP imaging, the time between CTP imaging and the end of the mechanical thrombecof the occlusion at 24 hours CT angiography.

\subsection{Preprocessing}

There is considerable variability in the acquisition protocol of CTP images and, due to the long acquisition time, the patient might move. During preprocessing, we account for both aspects, aiming to reduce the unnecessary variation that the network need to cope with. First, if the CTP was acquired with gantry tilt, the CTP images are resampled to an orthogonal grid that has the same $\mathrm{x}-\mathrm{y}$ plane and an orthogonalized $\mathrm{z}$-axis. Second, the CTP scan is motion corrected by rigidly aligning the volumes with the first volume. Registration is performed using Elastix, optimizing for the sum of squared differences. Finally, the images are spatially downsampled to $1.5 \times 1.5 \times 4 \mathrm{~mm}^{3}$ and temporally resampled to one image per two seconds. The rationale is that the original high spatial resolution might help the registrations, whereas for the perfusion analysis, which has a notoriously low Signal to Noise Ratio (SNR), a high resolution would only slow down processing. If the CTP was acquired in shuttle mode, i.e. with the patient continuously moving back and forth in the scanner resulting in variable scan times per CTP volume, we account for this and resample the time series such that each volume corresponds to a single time point.

\subsection{Network}

Our network is an extension of the model proposed by Kamnitsas et al. (2017) and has four different inputs. Each input is followed by series of operations such as convolutions and upsampling, called the pathway, and finally the outputs of the pathways are concatenated and fed into a common pathway that gives the voxelwise prediction. The first input is the CTP image which has 3 spatial dimensions plus time. This input is fed into a pathway consisting of 6 convolutional layers: three layers with 48 filters of $3 \times 3 \times 1$ and three layers with 64 filters of $3 \times 3 \times 3$. The second input is the downsampled CTP. The image is downsampled with a factor 3 in plane, resulting in voxels of $4.5 \times 4.5 \times 4 \mathrm{~mm}^{3}$ and which provides the network a wider spatial context to base its prediction on. This second input's pathway has the same architecture as the first one and is followed by an upsampling operation to recover the original resolution. The third input is the arterial input function (AIF), which is a one dimensional vector. This pathway consists of 2 convolution layers, each with 48 filters of $1 \times 1 \times 1$, and an upsampling operation. The last input is the metadata (1D) and this pathway only consists of an upsampling operation. At this point, the outputs of the four pathways have the same spatial dimensions and are concatenated in the feature dimension. This resulting output is fed through 3 convolutional layers with each a $1 \times 1 \times 1$ kernel. The first two have 150 filters and the final one has 1 filter and gives the prediction.

In all convolutional layers, we use batch normalization (Ioffe and Szegedy, 2015) and the PreLU activation function ( $\mathrm{He}$ et al. 2015).

This network has a fully convolutional structure, which allows to use dense inference and training. By predicting multiple adjacent voxels simultaneously, the computational efficiency is increased because redundant calculation in the overlapping receptive fields of the output neurons are avoided. We use an 


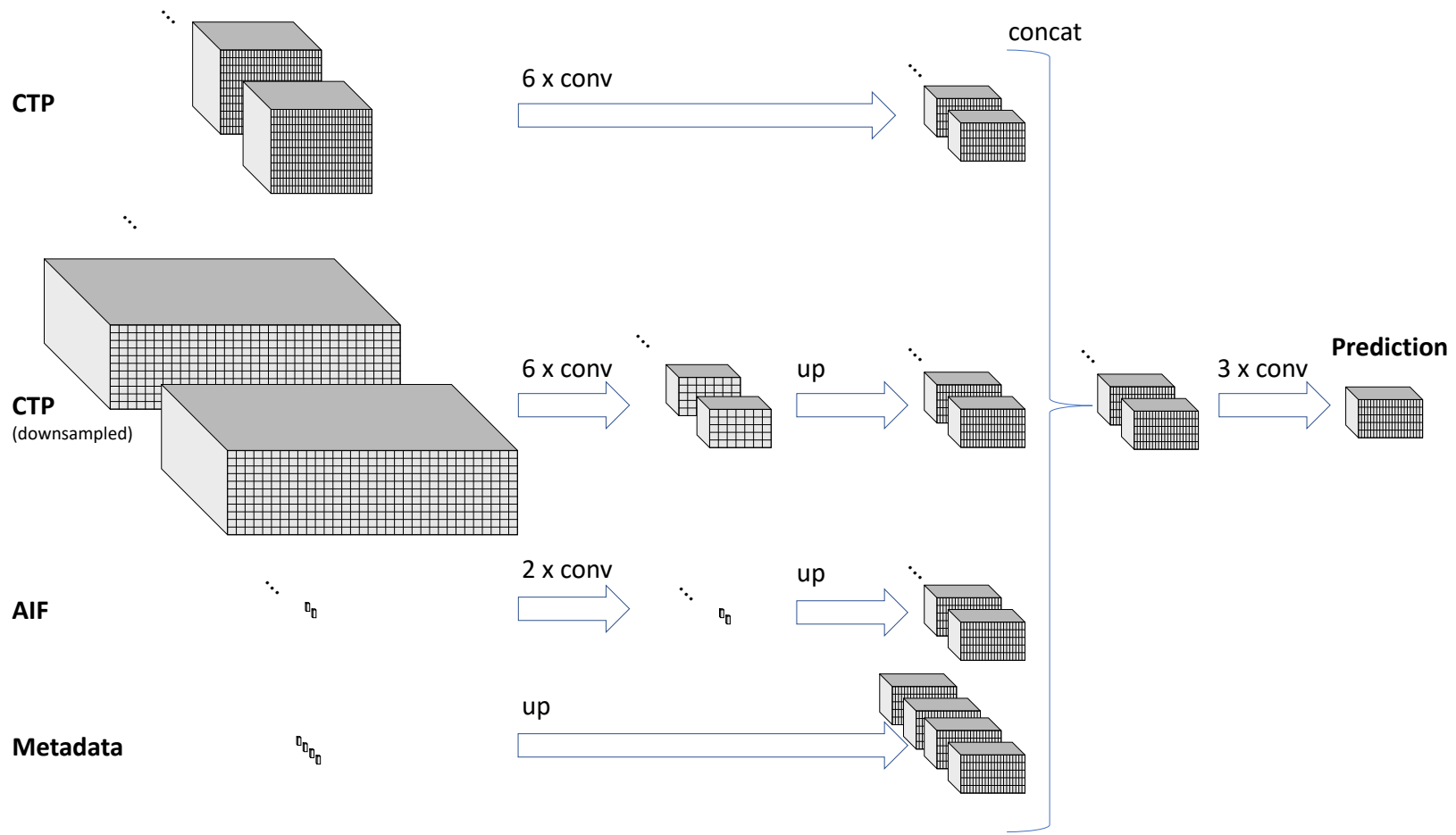

Figure 1: The proposed neural network. There are four inputs: the CTP (3D+time) the downsampled CTP (3D+time), the arterial input function (AIF, time) and the metadata (1D). The network has a fully convolutional structure, where each input can go through a series of convolutional layers before being upsampled to the original resolution. The outputs of the four pathways are concatenated and are passed through 3 convolutional layers with a 1x1x 1 kernel (acting as fully connected layers) before the prediction is given.

output size of $21 \times 21 \times 5$, giving the architecture visualized in Fig. 1]

The input intensities are normalized by first clipping them to the range of -100 to $1000 \mathrm{HU}$ and then linearly transforming them to have mean of zero and a standard deviation of one.

During training, the network is optimized by stochastic gradient descent with Nesterov momentum for the weighted crossentropy, with the positive class weighted ten times as heavy, resulting in balanced training. The training samples are uniformly chosen from within the intracranial volume and augmented by flipping samples left/right, small rotations, Gaussian noise and a CTP specific augmentation we introduced in (Robben and Suetens, 2018).

The CTP specific data augmentation exploits the fact that the perfusion measurements are a linear time invariant system. This means that, if the contrast injection was a bit later, both the AIF and the CTP timeseries would show the same delay. Similarly, if the injection was earlier, all curves would shift to the 305 left. However, this has no influence on the actual tissue perfusion status or the viability of the tissue. If the concentration of the iodine in the contrast agent were a fraction higher or lower, the concentration curves would change with the same fraction. But again, this has no impact on the tissue status. Hence, we $e_{310}$ augment our training dataset by applying a random time shift (earlier or later) and a random scaling of the attenuation variation. The shift is randomly chosen between -4 and 6 time points (since our measurements are discrete) and the scaling has a log normal distribution between with $\mu$ of 0 and $\sigma$ of 0.3 .

The method is implemented in Python using Keras and DeepVoxNet (Robben et al. 2018).

\subsection{Ablation study}

We want to measure the impact of the different design choices and hence we gradually ablate our method to understand how the different components contribute to the performance.

\section{The impact of no deconvolution}

To quantify the impact of the deconvolution-free approach we benchmark a network working on the deconvolved timeseries. To obtain the deconvolved time series, we use Tikhonov regularized SVD-based deconvolution (Fieselmann et al., 2011) with the Volterra discretization scheme (Sourbron et al., 2007) to which we simply refer as deconvolution. The deconvolution has one hyperparameter, the relative regularization parameter $\lambda_{\text {rel }}$ which determines the amount of filtering and which is set to 0.4. As the deconvolution is very noise sensitive, we first perform a spatial Gaussian smoothing with an isotropic sigma of $2.5 \mathrm{~mm}$. To make the comparison fair, we also test the proposed (i.e. the non-deconvolution based) network on this smoothed data - called Proposed (smoothed). The deconvolved time series are used as input to the network Proposed (deconvolved). This network and its training is identical to the one described in Section 2.2, with the exception that the AIF is no longer provided and the CTP specific data augmentation is turned off. 


\section{The impact of spatial context}

Current clinical systems do not take the spatial context into account to determine the tissue status of a voxel. We are interested in to what extent the spatial context affects the predictions. Hence we introduce two extra networks: One-voxel (smoothed) and One-voxel (deconvolved). Both only have three $1 \times 1 \times 1$ convolutions on the original resolution and do not use the subsampled version and hence do not use spatial context. Otherwise, both are identical to the earlier introduced methods.

\section{The impact of the CTP data augmentation}

We investigate the impact of our CTP specific data augmentation and train the proposed network also without the augmentation.

\section{The impact of the metadata}

Our network not only uses the imaging data, but also the treatment parameters. In current clinical practice, there is only a distinction between core and penumbra - roughly correspond- ${ }^{370}$ ing to immediate successful thrombectomy and no treatment at all. The additional treatment parameters our method uses might improve the accuracy of the predictions. To that end, we evaluate three variants of the proposed network. One that uses binarized mTICI scores (0-2a vs $2 \mathrm{~b}$ and 3 ), one that does not include ${ }^{375}$ time from onset to CTP and one that does not have time from CTP to end of thrombectomy.

\section{Experiments}

We evaluate the proposed method and its variants on the data of the MR CLEAN trial (described in more detail in the next section, Sec. 3.1p. In all experiments, we do a five-fold crossvalidation, and report the results aggregated over all the left-out subjects. The hyperparameters of the optimization are experimentally set based on preliminary experiments on the first fold. 385

\subsection{Dataset}

MR CLEAN was a multicenter study to investigate the benefit of thrombectomy in acute ischemic stroke (Berkhemer et al. 2015). The study's inclusion criteria are described in detail in the study protocol; in short: patients were randomized within 6 hours after stroke onset and when a large vessel occlusion was identified in the anterior circulation. In our work, we include all MR CLEAN subjects that had a baseline CTP of sufficient quality and a follow-up non-contrast computed tomography (NCCT) that could be registered to the acute CTP. This ${ }_{395}$ results in 188 included subjects. The selection process is shown in Fig. 2

The MR CLEAN study protocol prescribes follow-up imaging with NCCT at 24 hours and 5 days after onset. Where available, we use the 5 day follow-up otherwise the 24 hour follow- 400 up is used. The follow-up images were semi-automatically delineated and reviewed by an experienced reader (Boers et al. 2013, Bucker et al., 2017).

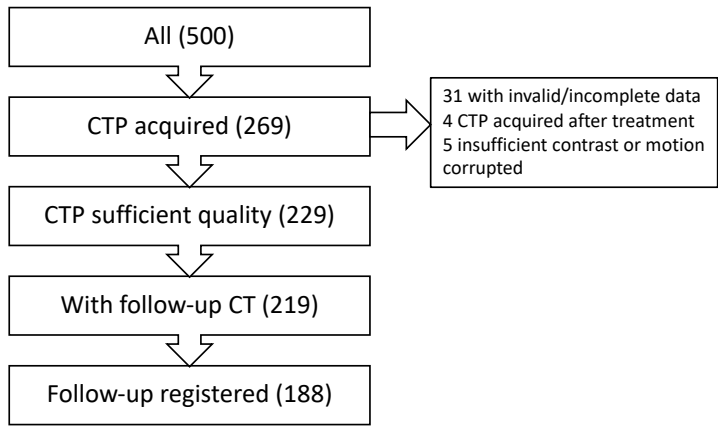

Figure 2: Subject selection.

The follow-up NCCT images are registered to the acute CTP images. First a rigid registration between the follow-up and the acute CTP is performed. Afterwards, a non-rigid registration is done in an attempt to compensate for brain swelling due to edema. The found transformations are applied to the final infarct delineations. Finally, we segment the cerebrospinal fluid (CSF) - in particular the ventricles - from the acute CTP images, and exclude those voxels from the transformed final infarct, under the reasoning that CSF cannot infarct and any overlap between CSF and final infarct is rather due to misalignment.

We manually select in each CTP image an arterial input function (AIF) and use those in all experiments. Hereto, we select the time series of the voxel that shows the largest increase in attenuation coefficent and is located in an unaffected proximal artery.

\subsection{Prediction of the final infarct}

The prediction of the final infarct is the most important capability of the network. For current clinical practice, the most important metric is the volume of the final infarct, and hence we focus on this metric and the difference in volume between the predicted volume and true volume. As predicted volume, we take the sum of all predicted probabilities, multiplied with the volume per voxel.

From a scientific point of view, the localization of the infarct is also interesting, and hence we also report the Dice and the soft Dice scores. The latter is like the standard Dice score, except that the predictions are not binarized. Non-parametric paired significance tests (bootstrapping) are used to test the significance of the results.

\subsection{Prediction of core and perfusion lesion}

It is interesting to see how the metadata influences the prediction of the network, in particular the difference between immediate recanalization and no recanalization. To this end, we predict for each subject in the test set the hypothetical final infarct volume in the case of early complete recanalization (mTICI 3 at 60 minutes) and in case of no recanalization at all. In the former scenario, the predicted final infarct should correspond to the infarct core at baseline imaging, whereas in the latter scenario, it should correspond to the perfusion lesion. 


\begin{tabular}{|c|c|c|c|}
\hline Method & $\begin{array}{c}\text { Mean } \\
\text { soft Dice }\end{array}$ & $\begin{array}{l}\text { Mean } \\
\text { Dice }\end{array}$ & $\begin{array}{c}\text { Mean absolute } \\
\text { volume error }(\mathrm{ml})\end{array}$ \\
\hline Proposed & $\mathbf{0 . 4 0}$ & $\overline{0.48}$ & 36.7 \\
\hline Proposed (smoothed) & $0.39 \quad(* *)$ & $0.45 \quad(* *)$ & 37.5 \\
\hline Proposed (deconvolved) & $0.36 \quad(* *)$ & $0.42 \quad(* *)$ & 39.1 \\
\hline One-voxel (smoothed) & $0.21 \quad(* *)$ & $0.15 \quad(* *)$ & 45.4 \\
\hline One-voxel (deconvolved) & $0.17 \quad(* *)$ & $0.04 \quad(* *)$ & $48.6 \quad(* *)$ \\
\hline Proposed (no data augmentation) & $0.39 \quad(*)$ & $0.46 \quad(* *)$ & 41.5 \\
\hline Proposed (binary mTICI) & 0.40 & 0.48 & $38.5 \quad(*)$ \\
\hline Proposed (no time from onset to CTP) & 0.40 & 0.48 & 38.4 \\
\hline $\begin{array}{l}\text { Proposed (no time from CTP to end of } \\
\text { thrombectomy) }\end{array}$ & $0.39 \quad(* *)$ & 0.47 & 41.1 \\
\hline
\end{tabular}

Table 1: The mean of the soft Dice, Dice and absolute volume error between the predicted and ground truth final infarct. A paired significance test is performed between the proposed method and its variants, with (*) indicating $P<0.05$ and (**) indicating $P<0.005$.
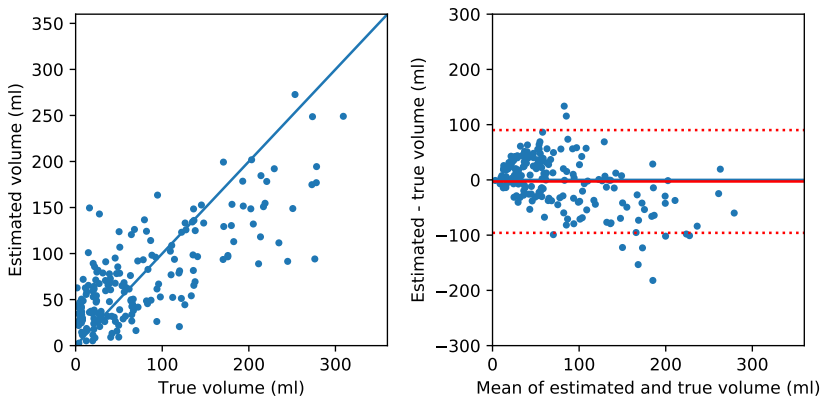

Figure 3: The true and predicted final infarct volumes for all subjects in the testing folds, using the proposed method.

\section{Results and discussion}

Fig. 3 shows a scatter plot and a Bland-Altman plot of the true and predicted volumes for all subjects in the testing folds using the proposed method. The mean volume error is $-2.8 \mathrm{ml}$ (the prediction is a slight underestimation) and the mean absolute volume error is $36.7 \mathrm{ml}$. The mean Dice score is 0.48 . A representative set of predictions is shown in Fig. 4.

The achieved Dice and volume errors seem respectively very low and high. We believe nevertheless that these are state-ofthe-art results on an inherently difficult problem. This is not an image segmentation task, where all the relevant information is the input, but a prediction problem with a ground truth that is defined on images acquired several days later. For example, the ISLES 2017 challenge (Winzeck et al., 2018) has a similar goal, namely predicting the final infarct from acute DWI and MR perfusion imaging, and reported a mean Dice 0.32 the best result. Of course, a direct comparison between these values is not possible since the ISLES challenge had different modalities (with especially the DWI imaging being very informative compared to perfusion imaging) and a different population (mostly early successful early recanalization, which results in small lesions and hence lower Dice scores), but ${ }_{465}$ it illustrates the difficulty of the problem.

\subsection{The impact of spatial context, no deconvolution and data augmentation}

Table 1 shows the mean Dice, soft Dice and absolute vol430 ume error of the proposed method and of the various ablated variants. It shows a gradual decrease in performance, both for removing the spatial context as for working on the deconvolved data. The beneficial effect of taking context into account is expected. Perfusion imaging is a noisy modality and suffers from artifacts which results in - often visually - erroneous predictions. In some clinical studies, the results given by the conventional voxelwise perfusion analysis are manually corrected. We hypothesize that a human rater is able to do so, because she takes spatial context into account. By providing spatial context, the network is able to do this as well, which greatly improves results: the mean Dice score more than triples and the volume error drops almost $20 \%$.

The benefit of working on the native data is more remarkable. It can be argued that, if we have a correct physical model, there is no need to re-learn that model a second time from our own limited training data. From the perspective of physics, deconvolution is the right way to approach the problem. However, the way that deconvolution should be regularized is not dictated by physics. We hypothesize that a neural network can do deconvolution better as it has a learned model of the noise and concentration curve shape. We showed this earlier on simulated data (Robben and Suetens, 2018) and see this now confirmed on real data: using the native data instead of the deconvolved data improved the mean Dice score significantly from 0.42 to 0.48 . Note however that learning this deconvolution implicitly is data-intensive and the proposed data augmentation is a necessary part, even with our relatively large training set.

Apart from the improved performance, a second advantage of avoiding the explicit deconvolution, is that the selection of the AIF can become part of the network. In this work, we used manually selected AIFs for all experiments, but AIF selection is a difficult problem and has great influence on the deconvolution results. However, if the AIF is an input to the network, it becomes possible to let the network learn to select the AIF from an input patch and have the AIF selection optimized in an end-to-end fashion, such as was explored by Hess et al. (2018). 

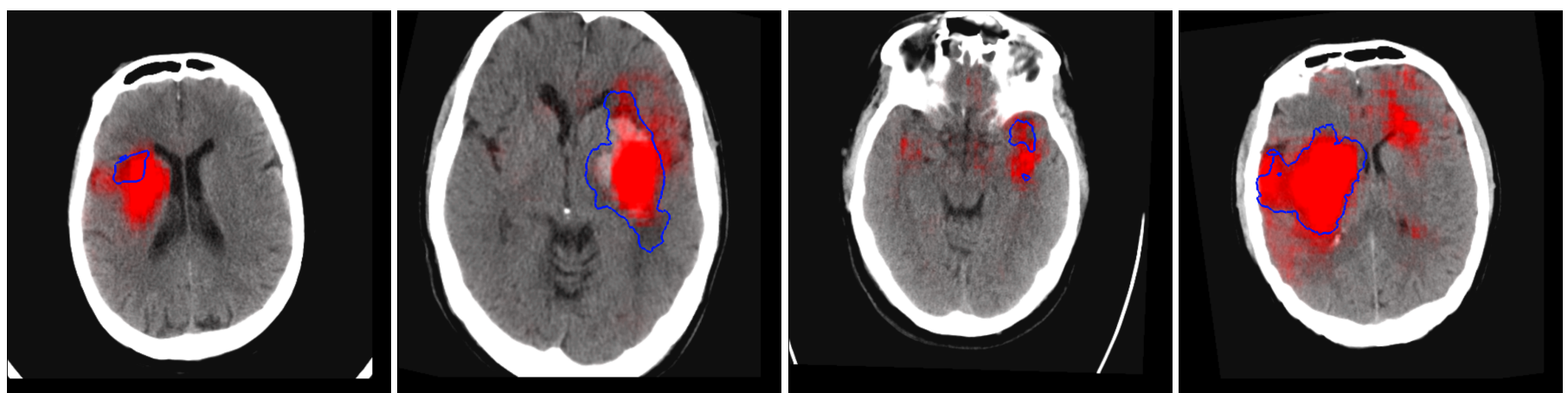

(a) Subjects with the $20 \%, 40 \%, 60 \%$ and $80 \%$ percentile Dice score in the subgroup with a true final infarct volume less than 100 ml.
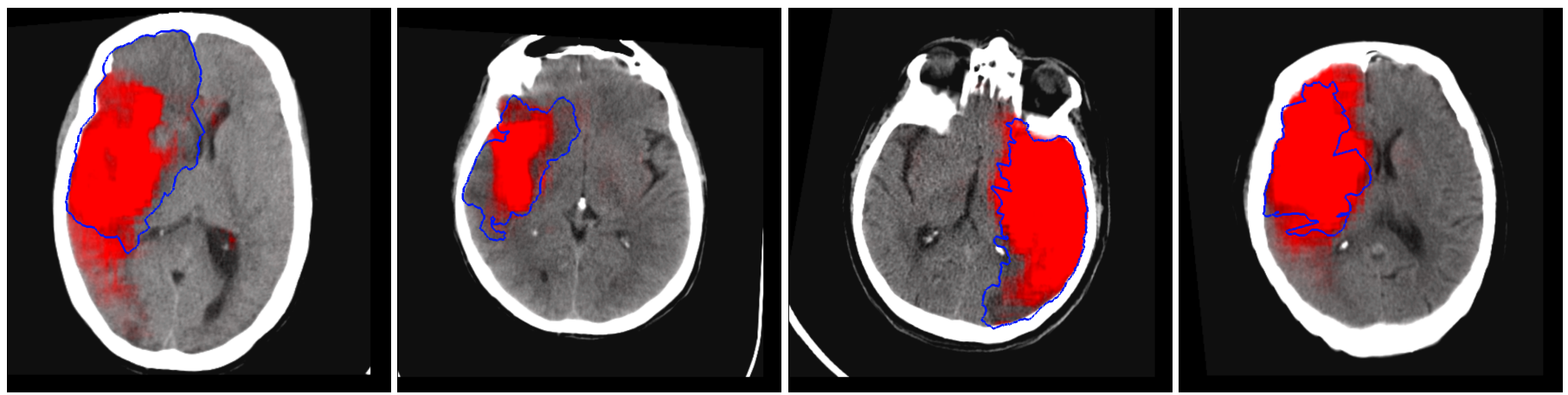

(b) Subjects with the $20 \%, 40 \%, 60 \%$ and $80 \%$ percentile Dice score in the subgroup with a true final infarct volume of at least $100 \mathrm{ml}$.

Figure 4: Predictions of the proposed method on a representative sample of subjects from the test set. The probabilistic predictions are overlayed in red on the follow-up NCCT scan whereas the ground truth final infarct is outlined in blue.

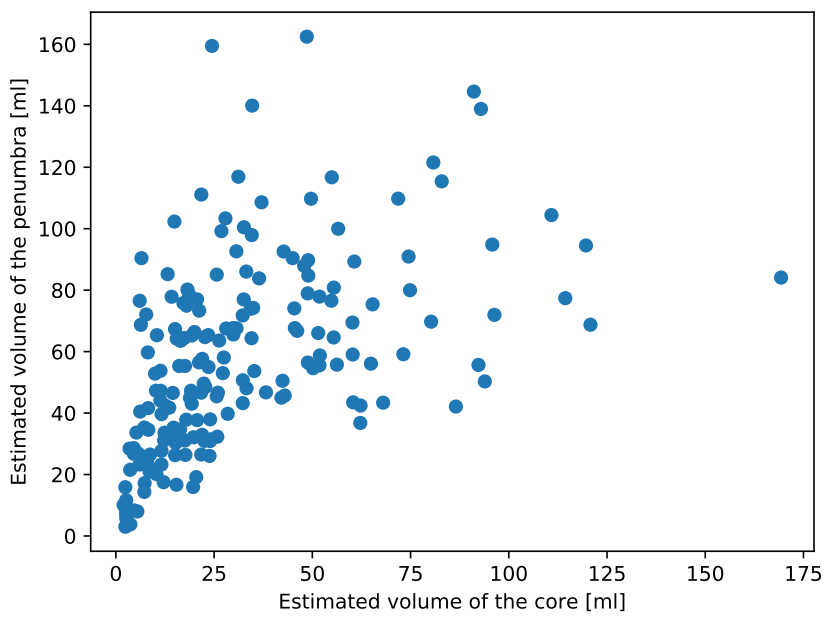

Figure 5: The predicted core and penumbra volumes for all subjects in the testing folds, using the proposed method. Green subjects have a target mismatch profile.

\subsection{The impact of the metadata}

Fig. 5 shows the volumes of the predicted core and penumbra (perfusion lesion minus core). It shows that the predicted final infarct volumes vary widely based on the treatment: the volume of the penumbra is the difference in final infarct volume between fast complete recanalization and no recanalization. This is as expected and already widely reported in literature.

In the ablation experiments, we also investigated the influence of the metadata on the quality of the predictions. Table 1 shows that in all cases the model became less predictive, which shows that the model effectively uses the metadata. Leaving out the time from CTP to end of treatment has the largest influence, increasing the mean absolute volume error with $12 \%$. Binarizing the mTICI and leaving out the time from onset to CTP have smaller but still significant effect, both increasing the error with $5 \%$. The importance of time from CTP to end of treatment and the precise mTICI scores were already earlier reported in literpature (Wheeler et al. 2015, D'Esterre et al., 2015, Kemmling et al., 2015). The influence of time from onset to CTP is more surprising, as earlier literature reported this does not have an influence (D'Esterre et al., 2015).

\subsection{The quality of the ground truth}

An important limitation of this study is the quality of the ground truth. First, follow-up imaging is always with NCCT, which is less sensitive than MR. Second, the follow-up NCCT scans are a mix of images acquired after 24 hours and after 5 
days. It is reported, on the MR CLEAN dataset, that the le-545 sion still grows after 24 hours: Bucker et al. (2017) report that between these time points the median infarct volume increases from $42 \mathrm{ml}$ to $64 \mathrm{ml}$ and more than half of the subjects witness more $30 \%$ relative growth. This means that there is variability on our ground truth, purely due to the moment of follow-up acquisition. Part of this volume increase is likely due to genuine delayed tissue death and part is due to edema, which is550 most pronounced at 3 to 5 days after the stroke. Finally, the brain edema increases the volume of the final infarct, and hence might result in overestimated core and penumbra volumes even though we tried to correct for this by non-rigid registration and555 CSF exclusion.

\subsection{Limited brain coverage}

Our dataset is acquired on a variety of scanners, and not all of ${ }^{560}$ them have full brain coverage during CTP acquisition. We find that the coverage along the axial direction is on average $65 \mathrm{~mm}$, with the first and third quartiles being 40 and $96 \mathrm{~mm}$. As a consequence, our ground truth final infarct volume is also limited ${ }^{566}$ to this field of view, resulting in an underestimation of the final infarct volume. The correlation between the final infarct volume within the CTP volume and the scan length is 0.16 .

\section{Conclusion}

We have shown that a neural network can learn to predict the ${ }_{575}$ final infarct volume from acute CTP images and the treatment parameters. This might help clinicians to evaluate the various treatment options. We performed a series of ablation experiments, which tested the contribution of the various components 580 of the method and showed the benefit of our deconvolution-free approach.

\section{Acknowledgements $\mathcal{E}$ disclosures}

David Robben is supported by an innovation mandate of Flanders Innovation \& Entrepreneurship (VLAIO) and is an employee of Icometrix. Anna M.M. Boers, Henk A Marquering, Yvo B.W.E.M. Roos and Charles B.L.M. Majoie ${ }^{590}$ are shareholders of Nico.lab. Lucianne L.C.M. Langezaal, Robert J. van Oostenbrugge and Paul Suetens report no disclosures. Wim H. van Zwam reports speaker fees from Stryker and Cerenovus paid to institution. Diederik W.J. Dippel re- ${ }^{595}$ ports funding from the Dutch Heart Foundation, Brain Foundation Netherlands, The Netherlands Organisation for Health Research and Development, Health Holland Top Sector Life Sciences \& Health, and unrestricted grants from AngioCare BV, Covidien/EV3®, MEDAC Gmbh/LAMEPRO, Penumbra Inc., Top Medical/Concentric, Stryker, Stryker European Operations BV, Medtronic, Thrombolytic Science, LLC for research, all paid to institution. Charles B.L.M. Majoie reports grants from ${ }^{605}$ European Commssion, CVON/Dutch Heart Founation, TWIN foundation, Stryker (paid to institution). Erasmus MC receives research grants from Stryker, Medtronic, Pernumbra, Siemens Healthineers, GE Healthcare and Philips Healthcare. Robin Lemmens is a senior clinical investigator of FWO Flanders.
The MR CLEAN trial was partly funded by the Dutch Heart Foundation and by unrestricted grants from AngioCare BV, Medtronic/Covidien/EV3®, MEDAC Gmbh/LAMEPRO, Penumbra Inc., Stryker®, and Top Medical/Concentric.

\section{References}

Albers, G.W., 2018. Late Window Paradox , 1-5doi 10.1161/STROKEAHA. 117.020200

Berkhemer, O.A., Fransen, P.S.S., Beumer, D., van den Berg, L.A., Lingsma, H.F., Yoo, A.J., Schonewille, W.J., Vos, J.A., Nederkoorn, P.J., Wermer, M.J.H., van Walderveen, M.A.A., Staals, J., Hofmeijer, J., van Oostayen, J.A., Lycklama à Nijeholt, G.J., Boiten, J., Brouwer, P.A., Emmer, B.J., de Bruijn, S.F., van Dijk, L.C., Kappelle, L.J., Lo, R.H., van Dijk, E.J., de Vries, J., de Kort, P.L.M., van Rooij, W.J.J., van den Berg, J.S.P., van Hasselt, B.A.A.M., Aerden, L.A.M., Dallinga, R.J., Visser, M.C., Bot, J.C.J., Vroomen, P.C., Eshghi, O., Schreuder, T.H., Heijboer, R.J.J., Keizer, K., Tielbeek, A.V., den Hertog, H.M., Gerrits, D.G., van den Berg-Vos, R.M., Karas, G.B., Steyerberg, E.W., Flach, H.Z., Marquering, H.A., Sprengers, M.E.S., Jenniskens, S.F.M., Beenen, L.F.M., van den Berg, R., Koudstaal, P.J., van Zwam, W.H., Roos, Y.B., van der Lugt, A., van Oostenbrugge, R.J., Majoie, C.B.L.M., Dippel, D.W.J., 2015. A Randomized Trial of Intraarterial Treatment for Acute Ischemic Stroke. New England Journal of Medicine 372, 11-20. doi 10.1056/NEJMoa1411587

Bivard, A., Levi, C., Spratt, N., Parsons, M., 2013. Perfusion CT in acute stroke: a comprehensive analysis of infarct and penumbra. Radiology 267, 543-550. doi 10.1148/radiol.12120971

Boers, A.M., Marquering, H.A., Jochem, J.J., Besselink, N.J., Berkhemer, O.A., Van Der Lugt, A., Beenen, L.F., Majoie, C.B., 2013. Automated cerebral infarct volume measurement in follow-up noncontrast CT scans of patients with acute ischemic stroke. American Journal of Neuroradiology 34, 1522-1527. doi 10.3174/ajnr.A3463

Boutelier, T., Kudo, K., Pautot, F., Sasaki, M., 2012. Bayesian hemodynamic parameter estimation by bolus tracking perfusion weighted imaging.

IEEE Transactions on Medical Imaging 31, 1381-1395. doi 10.1109/TMI . 2012.2189890

Bucker, A., Boers, A.M., Bot, J.C., Berkhemer, O.A., Lingsma, H.F., Yoo, A.J., van Zwam, W.H., van Oostenbrugge, R.J., van der Lugt, A., Dippel, D.W., Roos, Y.B., Majoie, C.B., Marquering, H.A., 2017. Associations of Ischemic Lesion Volume With Functional Outcome in Patients With Acute Ischemic Stroke. Stroke 48, 1233-1240. doi 10.1161/STROKEAHA.116. 015156

Christensen, S., Mouridsen, K., Wu, O., Hjort, N., Karstoft, H., Thomalla, G., Röther, J., Fiehler, J., Kucinski, T., Østergaard, L., 2009. Comparison of 10 Perfusion MRI Parameters in 97 Sub-6-Hour Stroke Patients Using VoxelBased Receiver Operating Characteristics Analysis. Stroke 40, 2055-2061. doi 10.1161/STROKEAHA.108.546069

D’Esterre, C.D., Boesen, M.E., Ahn, S.H., Pordeli, P., Najm, M., Minhas, P., Davari, P., Fainardi, E., Rubiera, M., Khaw, A.V., Zini, A., Frayne, R., Hill, M.D., Demchuk, A.M., Sajobi, T.T., Forkert, N.D., Goyal, M., Lee, T.Y., Menon, B.K., 2015. Time-dependent computed tomographic perfusion thresholds for patients with acute ischemic stroke. Stroke 46, 3390-3397. doi 10.1161/STROKEAHA.115.009250

Fahmi, F., Marquering, H.A., Streekstra, G.J., Beenen, L.F., Velthuis, B.K., VanBavel, E., Majoie, C.B., 2012. Differences in CT perfusion summary maps for patients with acute ischemic stroke generated by 2 software packages. American Journal of Neuroradiology 33, 2074-2080. doi 10.3174/ ajnr.A3110

Fieselmann, A., Kowarschik, M., Ganguly, A., Hornegger, J., Fahrig, R., 2011. Deconvolution-Based CT and MR Brain Perfusion Measurement: Theoretical Model Revisited and Practical Implementation Details. International Journal of Biomedical Imaging 2011, 1-20. doi 10.1155/2011/467563 arXiv: 467563

Guenego, A., Mlynash, M., Christensen, S., Kemp, S., Heit, J.J., Lansberg, M.G., Albers, G.W., 2018. Hypoperfusion ratio predicts infarct growth during transfer for thrombectomy. Annals of Neurology 84, 616-620. doi 10.1002 /ana. 25320

Hacke, W., Kaste, M., Bluhmki, E., Brozman, M., Dávalos, A., Guidetti, D., Larrue, V., Lees, K.R., Medeghri, Z., Machnig, T., Schneider, D., von Kummer, R., Wahlgren, N., Toni, D., 2008. Thrombolysis with Alteplase 3 to 4.5 
Hours after Acute Ischemic Stroke. New England Journal of Medicine 359, 1317-1329. doi 10.1056/NEJMoa0804656

615 He, K., Zhang, X., Ren, S., Sun, J., 2015. Delving Deep into Rectifiers: Surpassing Human-Level Performance on ImageNet Classification, in: 2015 IEEE International Conference on Computer Vision (ICCV), IEEE. pp. 1026-1034. doi 10.1109/ICCV.2015.123 arXiv:1502.01852

Hess, A., Meier, R., Kaesmacher, J., Jung, S., Scalzo, F., Liebeskind,690 D., Wiest, R., McKinley, R., 2018. Synthetic Perfusion Maps: Imaging Perfusion Deficits in DSC-MRI with Deep Learning. Arxiv , 111 arXiv: 1806.03848

Ho, K.C., El-Saden, S., Scalzo, F., Bui, A.A., Arnold, C.W., 2016. Predicting Acute Ischemic Stroke Tissue Fate Using Deep Learning on Source Perfu-695 sion MRI, in: International Stroke Conference.

Ioffe, S., Szegedy, C., 2015. Batch Normalization: Accelerating Deep Network Training by Reducing Internal Covariate Shift, in: Proceedings of the 32nd International Conference on Machine Learning, Lille, France. arXiv: 1502.03167

Kamnitsas, K., Ledig, C., Newcombe, V.F.J., Simpson, J.P., Kane, A.D., Menon, D.K., Rueckert, D., Glocker, B., 2017. Efficient multi-scale 3D CNN with fully connected CRF for accurate brain lesion segmentation. Medical Image Analysis 36, 61-78. doi 10.1016/j.media.2016.10. 004

Kemmling, A., Flottmann, F., Forkert, N.D., Minnerup, J., Heindel, W., Thomalla, G., Eckert, B., Knauth, M., Psychogios, M., Langner, S., Fiehler, J., 2015. Multivariate Dynamic Prediction of Ischemic Infarction and Tissue Salvage as a Function of Time and Degree of Recanalization. Journal of

1] Cerebral Blood Flow \& Metabolism 35, 1397-1405. doi 10.1038/jcbfm . 10 2015.144

Kleine, J.F., Kaesmacher, M., Wiestler, B., Kaesmacher, J., 2017. TissueSelective Salvage of the White Matter by Successful Endovascular Stroke Therapy. Stroke 48, 2776-2783. doi 10.1161/STROKEAHA.117.017903

Liebeskind, D.S., 2003. Collateral Circulation. Stroke 34, 2279-2284. doi 10. 15 1161/01.STR.0000086465.41263.06

McKinley, R., Häni, L., Gralla, J., El-Koussy, M., Bauer, S., Arnold, M., Fischer, U., Jung, S., Mattmann, K., Reyes, M., Wiest, R., 2017. Fully automated stroke tissue estimation using random forest classifiers (FASTER). Journal of Cerebral Blood Flow and Metabolism 37, 2728-2741. doi 10 . 1177/0271678X16674221

Meijs, M., Christensen, S., Lansberg, M.G., Albers, G.W., Calamante, F., 2016. Analysis of perfusion MRI in stroke: To deconvolve, or not to de-

1. convolve. Magnetic Resonance in Medicine 76, 1282-1290. doi 10.1002/ $\mathrm{mrm} .26024$

655 Nielsen, A., Hansen, M.B., Tietze, A., Mouridsen, K., 2018. Prediction of Tissue Outcome and Assessment of Treatment Effect in Acute Ischemic Stroke Using Deep Learning. Stroke 49, 1394-1401. doi 10.1161/STROKEAHA . 117.019740

Nogueira, R.G., Jadhav, A.P., Haussen, D.C., Bonafe, A., Budzik, R.F., Bhuva, P., Yavagal, D.R., Ribo, M., Cognard, C., Hanel, R.A., Sila, C.A., Hassan, A.E., Millan, M., Levy, E.I., Mitchell, P., Chen, M., English, J.D., Shah, Q.A., Silver, F.L., Pereira, V.M., Mehta, B.P., Baxter, B.W., Abraham, M.G., Cardona, P., Veznedaroglu, E., Hellinger, F.R., Feng, L., Kirmani, J.F., Lopes, D.K., Jankowitz, B.T., Frankel, M.R., Costalat, V., Vora, N.A., Yoo, A.J., Malik, A.M., Furlan, A.J., Rubiera, M., Aghaebrahim, A., Olivot, J.M., Tekle, W.G., Shields, R., Graves, T., Lewis, R.J., Smith, W.S., Liebeskind, D.S., Saver, J.L., Jovin, T.G., 2018. Thrombectomy 6 to 24 Hours after Stroke with a Mismatch between Deficit and Infarct. New England Journal of Medicine 378, 11-21. doi 10.1056/NEJMoa1706442

670 Pinto, A., Pereira, S., Meier, R., Alves, V., Wiest, R., Silva, C.A., Reyes, M., 2018. Enhancing Clinical MRI Perfusion Maps with Data-Driven Maps of Complementary Nature for Lesion Outcome Prediction, in: Frangi, A.F., Schnabel, J.A., Davatzikos, C., Alberola-López, C., Fichtinger, G. (Eds.), MICCAI, Springer International Publishing. pp. 107-115. doi 10.1007/ 978-3-030-00931-1_13 arXiv:1806.04413

Robben, D., Bertels, J., Willems, S., Vandermeulen, D., Maes, F., Suetens, P., 2018. DeepVoxNet: voxel-wise prediction for 3D images. Technical Report KUL/ESAT/PSI/1801. KU Leuven.

Robben, D., Suetens, P., 2018. Perfusion parameter estimation using neural networks and data augmentation, in: MICCAI SWITCH, Granada, Spain. arXiv: 1810.04898

Scalzo, F., Hao, Q., Alger, J.R., Hu, X., Liebeskind, D.S., 2012. Regional prediction of tissue fate in acute ischemic stroke. Annals of Biomed- ical Engineering 40, 2177-2187. doi 10.1007/s10439-012-0591-7 arXiv:NIHMS150003

Sourbron, S., Luypaert, R., Morhard, D., Seelos, K., Reiser, M., Peller, M., 2007. Deconvolution of bolus-tracking data: a comparison of discretization methods. Physics in Medicine and Biology 52, 6761-6778. doi 10.1088/ 0031-9155/52/22/014

Wheeler, H.M., Mlynash, M., Inoue, M., Tipirnini, A., Liggins, J., Bammer, R., Lansberg, M.G., Kemp, S., Zaharchuk, G., Straka, M., Albers, G.W., 2015. The Growth Rate of Early DWI Lesions is Highly Variable and Associated with Penumbral Salvage and Clinical Outcomes following Endovascular Reperfusion. International Journal of Stroke 10, 723-729. doi 10.1111/ijs.12436 arXiv: 15334406

Wintermark, M., Flanders, A.E., Velthuis, B., Meuli, R., Van Leeuwen, M., Goldsher, D., Pineda, C., Serena, J., Van Der Schaaf, I., Waaijer, A., Anderson, J., Nesbit, G., Gabriely, I., Medina, V., Quiles, A., Pohlman, S., Quist, M., Schnyder, P., Bogousslavsky, J., Dillon, W.P., Pedraza, S., 2006. Perfusion-CT assessment of infarct core and penumbra: Receiver operating characteristic curve analysis in 130 patients suspected of acute hemispheric stroke. Stroke 37, 979-985. doi 10.1161/01.STR.0000209238.61459. 39

Winzeck, S., Hakim, A., McKinley, R., Pinto, J.A.A.D.S.R., Alves, V., Silva, C., Pisov, M., Krivov, E., Belyaev, M., Monteiro, M., Oliveira, A., Choi, Y., Paik, M.C., Kwon, Y., Lee, H., Kim, B.J., Won, J.H., Islam, M., Ren, H., Robben, D., Suetens, P., Gong, E., Niu, Y., Xu, J., Pauly, J.M., Lucas, C., Heinrich, M.P., Rivera, L.C., Castillo, L.S., Daza, L.A., Beers, A.L., Arbelaezs, P., Maier, O., Chang, K., Brown, J.M., Kalpathy-Cramer, J., Zaharchuk, G., Wiest, R., Reyes, M., 2018. ISLES 2016 and 2017Benchmarking Ischemic Stroke Lesion Outcome Prediction Based on Mul-

tispectral MRI. Frontiers in Neurology 9. doi $10.3389 /$ fneur. 2018. 00679

Wu, O., Koroshetz, W.J., Ostergaard, L., Buonanno, F.S., Copen, W.a., Gonzalez, R.G., Rordorf, G., Rosen, B.R., Schwamm, L.H., Weisskoff, R.M., Sorensen, a.G., 2001. Predicting Tissue Outcome in Acute Human Cerebral Ischemia Using Combined Diffusion- and Perfusion-Weighted MR Imaging. Stroke 32, 933-942. doi 10.1161/01.STR.32.4.933 
${ }^{\star}$ Graphical Abstract

Acute ischemic stroke patient

Baseline CT perfusion

$+$

(Hypothetical) treatment parameters

\section{Prediction of final infarct}

Multi-scale CNN

- On the native measurements; without explicit deconvolution

- Trained and validated on multicenter dataset

- Improved performance
Immediate recanalization $78 \mathrm{ml}$

Follow-up NCCT after 5 days

Actual treatment (TICl 2A after 3h) $117 \mathrm{ml}$

vs.

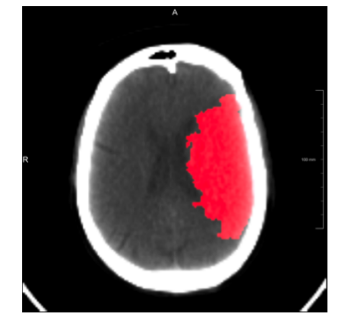

$121 \mathrm{ml}$
No recanalization

$141 \mathrm{ml}$ 
${ }^{*}$ Highlights

- prediction of the final infarct volume in acute ischemic stroke patients

- takes into account the baseline CTP images and the time and treatment parameters

- convolutional neural network trained and validated on a multicenter dataset

- operates on the native CTP measurements without explicit deconvolution

- improved performance over classical approach 


\section{${ }^{*}$ Conflict of Interest Form}

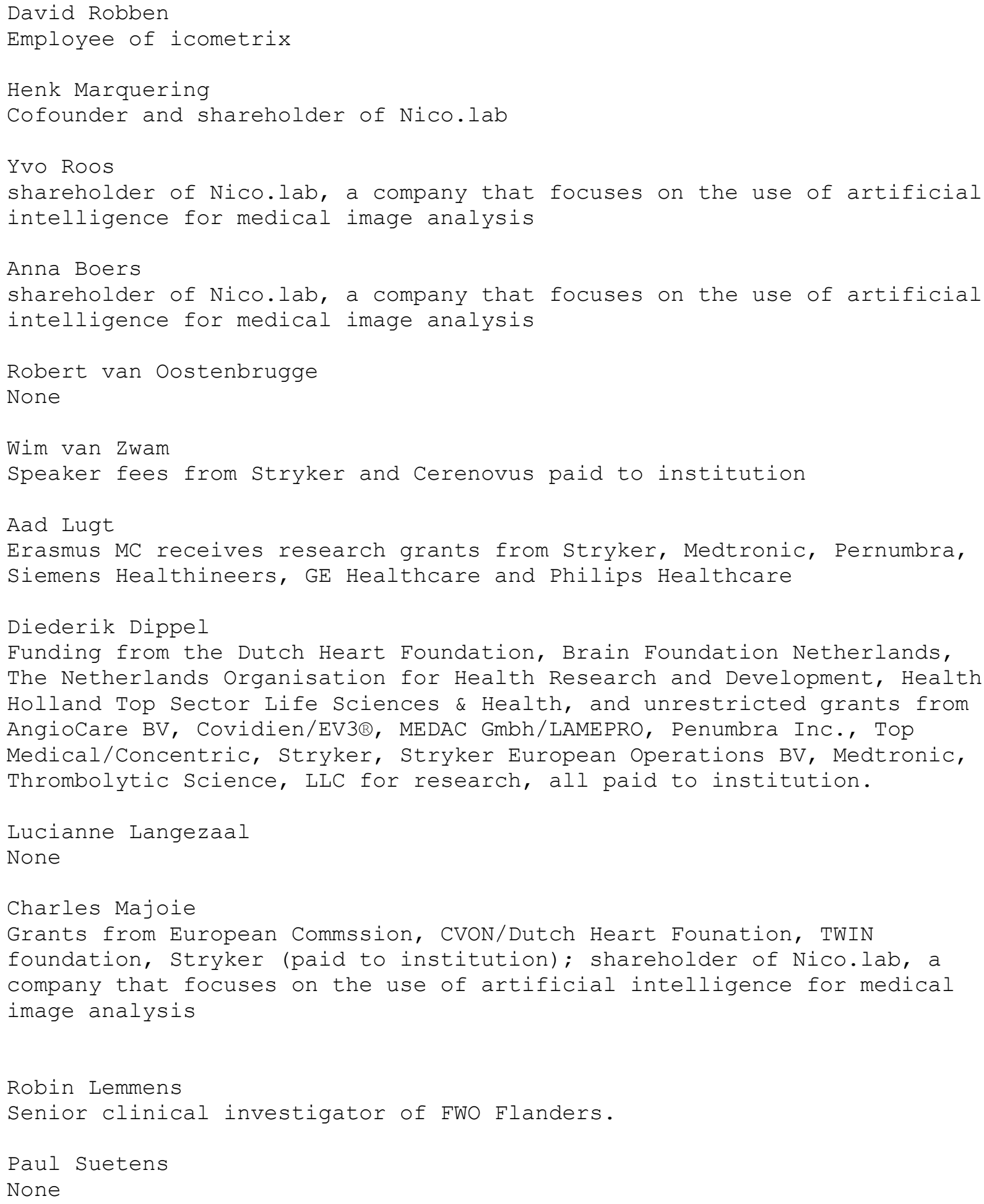

